\title{
Respostas da cultura do rabanete à inoculação de fungos solubilizadores de fosfatos ${ }^{(1)}$
}

\author{
Charles Narloch(2), Vetúria Lopes de Oliveira ${ }^{(3)}$, Jonas Ternes dos Anjos ${ }^{(4)}$ \\ e Germano Nunes Silva Filho(3)
}

\begin{abstract}
Resumo - O objetivo deste trabalho foi estudar o efeito dos fungos solubilizadores de fosfatos MSF-044, MSF-062 (Penicillium sp.) e MSF-087 (Aspergillus sp.) e da aplicação de fosfato solúvel $\left[\mathrm{Ca}\left(\mathrm{H}_{2} \mathrm{PO}_{4}\right)_{2} \cdot 2 \mathrm{H}_{2} \mathrm{O}\right]$, nas doses de $0,0,4,5,9,0,17,5,35,0,70,0$ e $140,0 \mathrm{mg} \mathrm{kg}^{-1}$ de $\mathrm{P}$, na produção de matéria seca e na absorção de fósforo pela cultura do rabanete. $\mathrm{O}$ experimento foi realizado em solo não-estéril, em casa de vegetação. Os isolados diferiram quanto à capacidade de promover a produção de matéria seca, dependendo da dose de P aplicada. Os isolados MSF-044 e MSF-062 foram mais eficientes sob baixas doses (4,5 a 17,5 $\mathrm{mg} \mathrm{kg}^{-1}$ de P), enquanto o isolado MSF-087 proporcionou maior produção de matéria seca somente na dose mais alta $\left(140,0 \mathrm{mg} \mathrm{kg}^{-1} \mathrm{de} \mathrm{P}\right)$. Plantas submetidas à inoculação do isolado MSF-062 (Penicillium sp.) e com 17,5 mg kg-1 de P apresentaram produção de matéria seca equivalente às obtidas por plantas com até 70,0 $\mathrm{mg}$ de $\mathrm{P}$ por $\mathrm{kg}$ de solo, sem inoculação. A quantidade total de $\mathrm{P}$ absorvida pelas plantas não foi influenciada pela inoculação, no solo, dos isolados testados.
\end{abstract}

Termos para indexação: Raphanus sativus, fósforo, solubilização, Aspergillus, Penicillium.

\section{Responses of radish culture to phosphate-solubilizing fungi}

\begin{abstract}
The objective of this work was to study the effect of phosphate-solubilizing fungi MSF-044, MSF-062 (Penicillium sp.) and MSF-087 (Aspergillus sp.) and soluble phosphate $\left[\mathrm{Ca}\left(\mathrm{H}_{2} \mathrm{PO}_{4}\right)_{2} \cdot 2 \mathrm{H}_{2} \mathrm{O}\right]$ at $0.0,4.5,9.0,17.5,35.0,70.0$ and $140.0 \mathrm{mg} \mathrm{kg}^{-1} \mathrm{P}$ on dry matter production and $\mathrm{P}$ uptake of radish, in non-sterilized soil, under green house conditions. Isolates differed in their capacity to stimulate dry matter production of plants, depending upon the P level. Isolates MSF-044 and MSF-062 were more efficient under low $\mathrm{P}$ (4.5 to $\left.17.5 \mathrm{mg} \mathrm{kg}^{-1} \mathrm{P}\right)$, while the isolate MSF-087 only stimulated dry matter production in the highest P level $\left(140.0 \mathrm{mg} \mathrm{kg}^{-1} \mathrm{P}\right)$. Plants inoculated with the isolate MSF-062, at $17.5 \mathrm{mg} \mathrm{kg}^{-1} \mathrm{P}$, presented no differences in dry matter compared to non-inoculated plants, with $70.0 \mathrm{mg} \mathrm{kg}^{-1} \mathrm{P}$. Phosphorus uptake by inoculated plants did not differ between treatments.
\end{abstract}

Index terms: Raphanus sativus, phosphorus, solubilization, Aspergillus, Penicillium.

\section{Introdução}

A maioria dos solos brasileiros apresenta altos teores de $\mathrm{P}$ total, mas a maior parte se encontra sob formas pouco solúveis, indisponíveis para os vegetais. A aplicação de fertilizantes fosfatados tem sido

\footnotetext{
(1) Aceito para publicação em 19 de julho de 2001.

(2) Universidade Federal de Santa Catarina (UFSC), Dep. de Microbiologia e Parasitologia (CCB), Caixa Postal 476, CEP 88040-900 Florianópolis, SC. Bolsista da Capes. E-mail: charles@narloch.com

(3) UFSC-CCB, Dep. de Microbiologia e Parasitologia. E-mail: veturia@mbox1.ufsc.br, germano@ccb.ufsc.br

(4) Universidade Regional de Blumenau, Dep. de Engenharia Florestal, Caixa Postal 1507, CEP 89010-971 Blumenau, SC. E-mail: anjos@furb.rct-sc.br
}

utilizada para suprir a deficiência de $\mathrm{P}$ do solo, porém uma parte considerável é convertida a compostos insolúveis de $\mathrm{Fe}$ e de $\mathrm{Al}$, em solos ácidos, ou de $\mathrm{Ca}$, em solos com reação neutra a alcalina (Raij, 1991).

Vários grupos de microrganismos apresentam capacidade de solubilizar fosfatos de rocha existentes ou adicionados no solo (Rodríguez \& Fraga, 1999; Whitelaw, 2000). Alguns microrganismos do solo ou da rizosfera podem, também, atuar sobre os fosfatos de baixa solubilidade formados após a aplicação de fosfatos solúveis, otimizando a eficiência da utilização do P (Chabot et al., 1996). A ação solubilizadora tem sido associada principalmente à produção de ácidos orgânicos (Sperber, 1958; Cunningham \& Kuiack, 1992; Bolan et al., 1994). A inoculação de microrganismos solubilizadores de fosfatos (MSF), 
aliados, ou não, a outros microrganismos benéficos do solo, pode aumentar a taxa de crescimento das plantas (Chabot et al., 1993; Kim et al., 1998; Singh \& Kapoor, 1999). A eficiente ação solubilizadora por fungos dos gêneros Aspergillus e Penicillium foi relatada por Illmer \& Schinner (1992) e Whitelaw (2000).

Por seu caráter intensivo, a olericultura é a atividade agrícola com a maior demanda de fertilizantes por unidade de área, os quais respondem por uma parcela considerável dos custos de produção. Isso justifica a adoção de práticas alternativas que possibilitem diminuir os custos, sem prejuízo à produtividade e à qualidade dos produtos. Com relação às crucíferas, a importância dos MSF é reforçada pela ausência de associações com fungos micorrízicos (Smith \& Read, 1997). A cultura do rabanete, embora pouco exigente em nutrientes, responde à adição de fertilizantes minerais, principalmente aos fosfatados. A facilidade de cultivo, aliada ao pequeno porte e ciclo curto, faz dessa cultura uma boa indicadora para estudos de fertilidade do solo, possibilitando a validação de suas respostas em estudos posteriores com outras espécies. O uso de inoculantes contendo MSF representa uma alternativa para aumentar a eficiência da adubação com fosfatos solúveis e diminuir os custos de produção.

O objetivo deste trabalho foi avaliar o efeito de fungos solubilizadores de fosfatos na produção de matéria seca e a absorção de $\mathrm{P}$ pela cultura do rabanete, sob diferentes doses de fosfato solúvel.

\section{Material e Métodos}

Foram utilizados três isolados de fungos solubilizadores de fosfatos, pertencentes à coleção do Laboratório de Microbiologia do Solo da Universidade Federal de Santa Catarina, previamente selecionados pela capacidade de solubilização de fosfatos naturais (Doyle et al., 1990) e identificados pelas siglas MSF-044, MSF-062 (Penicillium sp.) e MSF-087 (Aspergillus sp.).

A padronização do inóculo seguiu método adaptado de Oliveira (1988). Os isolados foram cultivados a $30^{\circ} \mathrm{C}$, durante sete dias, em cinco frascos contendo $50 \mathrm{~mL}$ de meio líquido glicose-extrato de levedura (GEL), modificado de
Sylvester-Bradley et al. (1982), previamente esterilizado. A cada frasco, adicionaram-se $10 \mathrm{~mL}$ de suspensão celular obtida pela lavagem de culturas dos isolados em meio sólido. Após a incubação, procedeu-se à eliminação do meio líquido e à deposição das culturas em frasco contendo $200 \mathrm{~mL}$ de água deionizada esterilizada. As culturas foram fragmentadas em um ultratriturador a $24.000 \mathrm{rpm}$ por cinco minutos, obtendo-se o inóculo líquido concentrado. $\mathrm{O}$ volume total foi redistribuído em três frascos esterilizados, que constituíram as repetições, e mantido sob refrigeração durante 72 horas.

Para quantificar e determinar a viabilidade do inóculo armazenado sob refrigeração e padronizar o volume a ser aplicado no ensaio com plantas, realizaram-se duas contagens de propágulos viáveis, a zero e 72 horas de armazenagem, utilizando-se o método de diluições sucessivas e contagem de colônias em meio GEL em placas de Petri, após incubação a $30^{\circ} \mathrm{C}$, durante 72 horas.

O experimento foi realizado em casa de vegetação, em um Cambissolo Háplico, coletado da camada de 0-15 cm de profundidade, em uma pequena propriedade de produção de hortaliças localizada no Município de Antônio Carlos, SC, em pastagem naturalizada. As características químicas e do teor de argila do solo, determinadas conforme Tedesco et al. (1995), é a seguinte: $\mathrm{pH}$ (água) 4,6; índice SMP 5,4; 4,1 mg dm${ }^{-3}$ de P; $>150 \mathrm{mg} \mathrm{dm}^{-3} \mathrm{de} \mathrm{K}^{+}$; $1,8 \mathrm{cmol}_{\mathrm{c}} \mathrm{dm}^{-3}$ de $\mathrm{Al}^{3+} ; 2,3 \mathrm{cmol}_{\mathrm{c}} \mathrm{dm}^{-3} \mathrm{de} \mathrm{Ca}^{2+}+\mathrm{Mg}^{2+}$; $36 \mathrm{~g} \mathrm{dm}^{-3}$ de matéria orgânica, e $200 \mathrm{~g} \mathrm{dm}^{-3}$ de argila.

Trinta dias antes do plantio, o solo foi passado em peneira com abertura de $2 \mathrm{~mm}$ e corrigido quanto à acidez com 3,25 g kg-1 de uma mistura de $\mathrm{CaCO}_{3}$ e $\mathrm{MgCO}_{3}(2: 1)$, para elevar o pH (água) a 6,0 (Comissão de Fertilidade do Solo RS/SC, 1989). Vasos contendo $500 \mathrm{~g}$ de solo, sem qualquer tratamento de desinfecção, receberam $100 \mathrm{mg} \mathrm{kg}^{-1}$ de $\mathrm{K}$ e $200 \mathrm{mg} \mathrm{kg}^{-1}$ de $\mathrm{N}$. Com base em curva de resposta obtida em experimento preliminar, foram definidas as doses de fosfato monocálcico $\left[\left(\mathrm{Ca}\left(\mathrm{H}_{2} \mathrm{PO}_{4}\right)_{2} \cdot 2 \mathrm{H}_{2} \mathrm{O}\right.\right.$ p.a.] a serem testadas $\left(0,0,4,5,9,0,17,5,35,0,70,0\right.$ e $140 \mathrm{mg} \mathrm{kg}^{-1}$ de P). Avaliaram-se, ainda, quatro tratamentos de inoculação (contendo isoladamente os fungos MSF-044, MSF-062, MSF-087 e uma testemunha sem inoculação), constituindo um experimento em fatorial $7 \times 4$. Os tratamentos foram dispostos em delineamento completamente casualizado, com três repetições.

O volume do inóculo líquido aplicado foi de $10^{9}$ propágulos por kg de solo. O inóculo, os nutrientes e o 
solo foram homogeneizados e, em seguida, cada vaso foi semeado com seis sementes de rabanete (Raphanus sativus L. cv. Crimson Giant). Após a emergência, foram mantidas duas plantas por vaso. Durante o período experimental, o teor de umidade do solo foi mantido em $80 \%$ da capacidade máxima de retenção de água do solo. A temperatura variou entre 13 e $23^{\circ} \mathrm{C}$, e obtiveram-se, em média, 14 horas de luz por dia. Trinta dias após a semeadura, as plantas foram colhidas, lavadas e secadas em estufa a $75^{\circ} \mathrm{C}$, para avaliação do peso da matéria seca e determinação do teor de $\mathrm{P}$ no tecido, conforme Tedesco et al. (1995). Com base nos dados de produção de matéria seca e da concentração de P na planta, determinou-se a quantidade do nutriente absorvida. Os dados foram submetidos à análise de variância. As médias dos fatores analisados e das interações entre eles foram comparadas pelo teste de Duncan a $5 \%$ de probabilidade.

\section{Resultados e Discussão}

Houve interação entre as doses de $\mathrm{P}$ e os isolados inoculados tanto na produção de matéria seca (Tabela 1) quanto na quantidade total de P absorvido pelas plantas de rabanete (Tabela 2).

Nos tratamentos sem adição de $P$, não houve efeito da inoculação sobre ambas as variáveis (Tabelas 1 e 2), o que demonstra que os isolados não atuaram na solubilização de fosfatos nativos do solo. A inoculação dos isolados MSF-044 e MSF-062 (Penicillium sp.), associada à aplicação de doses mais baixas de $\mathrm{P}\left(4,5\right.$ a $\left.17,5 \mathrm{mg} \mathrm{kg}^{-1} \mathrm{de} \mathrm{P}\right)$, proporcio-

Tabela 1. Produção de matéria seca (mg/planta) de rabanete cultivar Crimson Giant, cultivado em solo com diferentes doses de fosfato de cálcio $\left[\mathrm{Ca}\left(\mathrm{H}_{2} \mathrm{PO}_{4}\right)_{2} \cdot 2 \mathrm{H}_{2} \mathrm{O}\right] \mathrm{e}$ submetido à inoculação de fungos solubilizadores de fosfatos: Penicillium spp. (isolados MSF-044 e MSF-062) e Aspergillus sp. (isolado MSF-087). Médias de três repetições $^{(1)}$

\begin{tabular}{ccllcl}
\hline $\mathrm{P}\left(\mathrm{mg} \mathrm{kg}^{-1}\right)$ & Testemunha & MSF-044 & MSF-062 & MSF-087 & Média \\
\hline 0,0 & $157 \mathrm{a}$ & $189 \mathrm{a}$ & $163 \mathrm{a}$ & $158 \mathrm{a}$ & $167 \mathrm{~A}$ \\
4,5 & $346 \mathrm{~b}$ & $420 \mathrm{bc}$ & $484 \mathrm{~cd}$ & $390 \mathrm{bc}$ & $410 \mathrm{~B}$ \\
9,0 & $436 \mathrm{bc}$ & $549 \mathrm{de}$ & $612 \mathrm{e}$ & $487 \mathrm{~cd}$ & $521 \mathrm{C}$ \\
17,5 & $630 \mathrm{e}$ & $620 \mathrm{ef}$ & $719 \mathrm{fg}$ & $625 \mathrm{ef}$ & $648 \mathrm{D}$ \\
35,0 & $794 \mathrm{ghij}$ & $769 \mathrm{gh}$ & $791 \mathrm{ghi}$ & $795 \mathrm{ghij}$ & $787 \mathrm{E}$ \\
70,0 & $810 \mathrm{ghij}$ & $823 \mathrm{ghij}$ & $786 \mathrm{ghi}$ & $845 \mathrm{hij}$ & $816 \mathrm{E}$ \\
140,0 & $900 \mathrm{jl}$ & 9671 & $883 \mathrm{ijl}$ & $1.006 \mathrm{~m}$ & $939 \mathrm{~F}$ \\
\hline Média & $582 \mathrm{~A}$ & $620 \mathrm{~A}$ & $634 \mathrm{~A}$ & $615 \mathrm{~A}$ & \\
\hline
\end{tabular}

${ }^{(1)}$ Médias das interações seguidas da mesma letra não diferem entre si pelo teste de Duncan a 5\% de probabilidade. nou maior produção de matéria seca pelas plantas em relação à testemunha (Tabela 1). Esse efeito foi obtido pelas plantas que receberam o isolado MSF-062, nos tratamentos de 4,5 a 17,5 $\mathrm{mg} \mathrm{kg}^{-1} \mathrm{de} \mathrm{P}$, e o isolado MSF-044, no tratamento de $9,0 \mathrm{mg} \mathrm{kg}^{-1}$ de P. Incrementos na produção de matéria seca pelas plantas com inoculação desses isolados foram também observados com o aumento da dose de $\mathrm{P}$ de 4,5 para 9,0 $\mathrm{mg} \mathrm{kg}^{-1}$ de P; não ocorreu o mesmo com as plantas sem inoculação. Na dose de $9,0 \mathrm{mg} \mathrm{kg}^{-1}$ de $\mathrm{P}$, ambos os isolados proporcionaram uma produção de matéria seca equivalente à obtida pela aplicação de $17,5 \mathrm{mg} \mathrm{kg}^{-1}$ de $\mathrm{P}$, na testemunha. Na dose de $17,5 \mathrm{mg} \mathrm{kg}^{-1}$ de $\mathrm{P}$, a produção de matéria seca obtida pelas plantas que receberam o isolado MSF-062 foi equivalente à obtida pela aplicação de até $70,0 \mathrm{mg} \mathrm{kg}^{-1} \mathrm{de} \mathrm{P}$, na testemunha.

O isolado MSF-087 (Aspergillus sp.) proporcionou maior produção de matéria seca somente na dose de $140,0 \mathrm{mg} \mathrm{kg}^{-1}$ de $\mathrm{P}$, quando comparada à testemunha (Tabela 1).

Quando analisada como fator isolado, a inoculação não promoveu diferenças na quantidade total de $\mathrm{P}$ absorvido pelas plantas (Tabela 2). A única exceção foram as plantas com inoculação do isolado MSF-087 (Aspergillus sp.) na dose de $35,0 \mathrm{mg} \mathrm{kg}^{-1}$ de $\mathrm{P}$, que proporcionaram resultados inferiores aos da testemunha.

A maior eficiência na produção de matéria seca de plantas com inoculação de MSF e fertilizadas com fosfatos solúveis tem sido atribuída à atuação dos

Tabela 2. Quantidade total de P absorvido (mg/planta) pelo rabanete cultivar Crimson Giant, cultivado em solo com diferentes doses de fosfato de cálcio $\left[\mathrm{Ca}\left(\mathrm{H}_{2} \mathrm{PO}_{4}\right)_{2} \cdot 2 \mathrm{H}_{2} \mathrm{O}\right]$ e submetido à inoculação de fungos solubilizadores de fosfatos: Penicillium spp. (isolados MSF-044 e MSF-062) e Aspergillus sp. (isolado MSF-087). Médias de três repetições $^{(1)}$.

\begin{tabular}{ccllll}
\hline $\mathrm{P}\left(\mathrm{mg} \mathrm{kg}^{-1}\right)$ & Testemunha & MSF-044 & MSF-062 & MSF-087 & Média \\
\hline 0,0 & $0,22 \mathrm{a}$ & $0,27 \mathrm{a}$ & $0,21 \mathrm{a}$ & $0,22 \mathrm{a}$ & $0,23 \mathrm{~A}$ \\
4,5 & $0,50 \mathrm{ab}$ & $0,55 \mathrm{ab}$ & $0,91 \mathrm{bcd}$ & $0,56 \mathrm{ab}$ & $0,63 \mathrm{AB}$ \\
9,0 & $0,73 \mathrm{bc}$ & $1,04 \mathrm{cde}$ & $1,14 \mathrm{cdef}$ & $0,83 \mathrm{bcd}$ & $0,93 \mathrm{~B}$ \\
17,5 & $1,40 \mathrm{ef}$ & $1,46 \mathrm{ef}$ & $1,51 \mathrm{f}$ & $1,20 \mathrm{def}$ & $1,39 \mathrm{C}$ \\
35,0 & $2,39 \mathrm{hi}$ & $2,22 \mathrm{gh}$ & $2,38 \mathrm{hi}$ & $1,94 \mathrm{~g}$ & $2,23 \mathrm{D}$ \\
70,0 & $2,72 \mathrm{ij}$ & $2,92 \mathrm{jl}$ & $2,76 \mathrm{ij}$ & $3,07 \mathrm{jl}$ & $2,87 \mathrm{E}$ \\
140,0 & $3,301 \mathrm{~m}$ & $3,56 \mathrm{~m}$ & $3,66 \mathrm{~m}$ & $3,69 \mathrm{~m}$ & $3,55 \mathrm{~F}$ \\
\hline Média & $1,61 \mathrm{~A}$ & $1,72 \mathrm{~A}$ & $1,80 \mathrm{~A}$ & $1,64 \mathrm{~A}$ \\
\hline \multicolumn{7}{c}{} \\
(1) Médias das interações seguidas da mesma letra não diferem entre si pelo \\
teste de Duncan a 5\% de probabilidade.
\end{tabular}

Pesq. agropec. bras., Brasília, v. 37, n. 6, p. 841-845, jun. 2002 
microrganismos sobre os fosfatos de baixa solubilidade, formados no solo após a adição desses fertilizantes (Chabot et al., 1996). O mecanismo envolvido nesse processo é a ação direta de ácidos orgânicos produzidos pelos MSF, ou a ação quelante dos ácidos sobre os cátions acompanhantes dos fosfatos (Whitelaw, 2000).

Além da solubilização, muitos MSF produzem substâncias promotoras do crescimento de plantas que estimulam o maior rendimento das culturas que receberam inóculo de MSF (Gonzales-Eguiarte \& Barea, 1975; Leinhos \& Vacek, 1994). A ausência de valores significativos nas quantidades de $\mathrm{P}$ absorvido (Tabela 2), aliada à maior produção de matéria seca (Tabela 1) nos tratamentos inoculados e com baixas doses de $\mathrm{P}$, sugere que os fungos testados neste estudo possam produzir hormônios vegetais ou outras substâncias promotoras do crescimento das plantas, o que deve ser avaliado em ensaios posteriores. Freitas et al. (1997) também obtiveram maior crescimento e rendimento de canola (Brassica napus L.) com inoculação de bactérias solubilizadoras de fosfatos, sem qualquer efeito sobre a quantidade de $\mathrm{P}$ absorvido.

A maior produção de matéria seca nas doses mais baixas de $\mathrm{P}$ aplicado (4,5 a 17,5 $\mathrm{mg} \mathrm{kg}^{-1} \mathrm{de} \mathrm{P}$ ), nos tratamentos com inoculação dos isolados MSF-044 e MSF-062 (Penicillium sp.) (Tabela 1), é semelhante à obtida por Gonzales-Eguiarte \& Barea (1975) em relação ao crescimento e absorção de $\mathrm{P}$ pelo tomateiro. Os autores sugerem que os MSF têm a atividade solubilizadora inibida na presença de $\mathrm{P}$ solúvel. $\mathrm{O}$ aumento da disponibilidade de $\mathrm{P}$ solúvel, a partir de determinada concentração, pode inibir a liberação dos ácidos orgânicos responsáveis pela solubilização do P inorgânico, bem como a ação das fosfatases, responsáveis pela mineralização do $\mathrm{P}$ orgânico do solo (Nahas et al., 1994). Comportamento semelhante ocorre com os fungos micorrízicos, nos quais a ação solubilizadora, a intensidade de colonização radicular e o efeito sobre o crescimento diminuem com o aumento do P disponível (Lapeyrie et al., 1991).

Por outro lado, o isolado MSF-087 (Aspergillus sp.) apresentou comportamento contrário, proporcionando maior produção de matéria seca do rabanete somente na dose de $140 \mathrm{mg} \mathrm{kg}^{-1}$ de P
(Tabela 1). Em meio de cultura, Silva Filho (1998) constatou que os fungos do gênero Aspergillus, incluindo o isolado em questão, quando comparados aos do gênero Penicillium, apresentam maior crescimento em placas de Petri, mas menor eficiência na solubilização de fosfatos. Isso explicaria, neste estudo, a ausência de efeito significativo da inoculação do isolado MSF-087 sobre a quantidade de P absorvido, para as doses de até $17,5 \mathrm{mg} \mathrm{kg}^{-1} \mathrm{de} \mathrm{P}$, quando comparadas à testemunha (Tabela 2). O P solubilizado por esse isolado nas doses mais baixas de fertilizante aplicadas pode ter sido imobilizado pelo fungo durante sua multiplicação no solo, hipótese esta que é reforçada pela quantidade de $\mathrm{P}$ absorvido na dose de $35 \mathrm{mg} \mathrm{kg}^{-1}$ de $\mathrm{P}$, a qual foi inferior à da testemunha. Neste caso, a atuação do isolado só pôde ser percebida na dose mais alta de $\mathrm{P}, 140 \mathrm{mg} \mathrm{kg}^{-1}$ (Tabela 1).

\section{Conclusões}

1. Os isolados MSF-044, MSF-062 (Penicillium sp.) e MSF-087 (Aspergillus sp.) diferem quanto à capacidade de promover a produção de matéria seca e a absorção de P pela cultura do rabanete, atuando os primeiros sob as doses mais baixas de fosfato adicionadas (4,5 a 17,5 $\left.\mathrm{mg} \mathrm{kg}^{-1} \mathrm{de} \mathrm{P}\right)$, e o último, sob dose mais alta de $\mathrm{P}\left(140 \mathrm{mg} \mathrm{kg}^{-1}\right)$.

2. Nas doses mais baixas de $\mathrm{P}\left(4,5\right.$ a $\left.17,5 \mathrm{mg} \mathrm{kg}^{-1}\right)$, os isolados MSF-044 e MSF-062 (Penicillium sp.) apresentam maior eficiência na produção de matéria seca da cultura do rabanete, em relação à testemunha.

3. Na maior dose de $\mathrm{P}\left(140 \mathrm{mg} \mathrm{kg}^{-1}\right)$, o isolado MSF-087 é mais eficiente na produção de matéria seca da cultura do rabanete, em relação à testemunha.

\section{Referências}

BOLAN, N. S.; NAIDU, R.; MAHIMAIRAJA, S.; BASKARAN, S. Influence of low molecular-weight organic acids on the solubilization of phosphates. Biology and Fertility of Soils, New York, v. 18, p. 311-319, 1994.

CHABOT, R.; ANTOUN, H.; CESCAS, M. P. Growth promotion of maize and lettuce by phosphate-solubilizing Rhizobium leguminosarum biovar. phaseoli. Plant and Soil, Dordrecht, v. 184, p. 311-321, 1996. 
CHABOT, R.; ANTOUN, H.; CESCAS, M. P. Stimulation de la croissance du maïs et de la laitue romaine par des microorganismes dissolvant le phosphore inorganique. Canadian Journal of Microbiology, Ottawa, v. 39, p. 941-947, 1993.

COMISSÃO DE FERTILIDADE DO SOLO RS/SC (Passo Fundo, RS). Recomendações de adubação e calagem para os estados do Rio Grande do Sul e Santa Catarina. 2. ed. Passo Fundo: Sociedade Brasileira de Ciência do Solo/Embrapa-CNPT, 1989. 128 p.

CUNNINGHAM, J. E.; KUIACK, C. Production of citric and oxalic acids and solubilization of calcium phosphate by Penicillium bilajii. Applied and Environmental Microbiology, New York, v. 58, p. 1451-1458, 1992.

DOYLE, L. G. M.; SCHARF, R.; SILVA FILHO, G. N. Avaliação da população e do potencial de microorganismos solubilizadores de fosfatos de solos cultivados com fruteiras temperadas de Santa Catarina. Biotemas, Florianópolis, v. 3, n. 2, p. 59-76, 1990.

FREITAS, J. R.; BANERJEE, M. R.; GERMIDA, J. J. Phosphate-solubilizing rhizobacteria enhance the growth and yield but not phosphorus uptake of canola (Brassica napus L.). Biology and Fertility of Soils, New York, v. 24, p. 358-364, 1997.

GONZALES-EGUIARTE, D.; BAREA, J. M. Fertilización biológica con fosfobacterias productoras de fitohormonas en suelos deficientes en P: influencia de la adición de P y materia orgánica. Revista Latinoamericana de Microbiología, México, v. 17, p. 227-232, 1975.

ILLMER, P.; SCHINNER, F. Solubilization of inorganic phosphates by microorganisms isolated from forest soils. Soil Biology and Biochemistry, London, v. 24, p. 389395, 1992.

KIM, K. Y.; JORDAN, D.; McDONALD, G. A. Effect of phosphate-solubilizing bacteria and vesicular-arbuscular mycorrhizae on tomato growth and soil microbial activity. Biology and Fertility of Soils, New York, v. 26, p. 7987, 1998.

LAPEYRIE, F.; RANGER, J.; VAIRELLES, D. Phosphate solubilizing activity of ectomycorrhyzal fungi in vitro. Canadian Journal of Botany, Ottawa, v. 69, n. 2, p. 342346, 1991.

LEINHOS, V.; VACEK, O. Biosynthesis of auxins by phosphate-solubilizing rhizobacteria from wheat (Triticum aestivum) and rye (Secale cereale). Microbiological Research, Jena, v. 149, p. 31-35, 1994.

NAHAS, E.; CENTURION, J. F.; ASSIS, L. C. Microrganismos solubilizadores de fosfatos e produtores de fosfatases de vários solos. Revista Brasileira de Ciência do Solo, Campinas, v. 18, p. 43-48, 1994.

OLIVEIRA, V. L. Interactions entre les microorganismes du sol et l'établissement de la symbiose ectomycorhizienne chez le hêtre (Fagus silvatica $\mathbf{L}$.) avec Hebeloma crustuliniforme (Bull. Ex Saint-Amans) Quél. et Paxillus involutus Batsch. Ex Fr. 1988. 118 p. Thèse (Doctorat) - Institut National de la Recherche Agronomique, Nancy.

RAIJ, B. van. Fertilidade do solo e adubação. Piracicaba: Ceres/Potafos, 1991. 343 p.

RODRÍGUEZ, H.; FRAGA, R. Phosphate solubilizing bacteria and their role in plant growth promotion. Biotechnology Advances, Oxford, v. 17, p. 319-339, 1999.

SILVA FILHO, G. N. Solubilização de fosfatos pela microbiota do solo. 1998. $140 \mathrm{f}$. Tese (Doutorado) - Universidade Federal do Rio Grande do Sul, Porto Alegre.

SINGH, S.; KAPOOR, K. K. Inoculation with phosphatesolubilizing microorganisms and a vesicular arbuscular mycorrhizal fungus improves dry matter yield and nutrient uptake by wheat grown in a sandy soil. Biology and Fertility of Soils, New York, v. 28, p. 139-144, 1999.

SMITH, S. E.; READ, D. J. Mycorrhizal symbiosis. 2. ed. Cambridge, Inglaterra: Academic Press, 1997. 605 p.

SPERBER, J. I. Solution of apatite by soil microorganisms producing organic acids. Australian Journal of Agricultural Research, Melbourne, v. 9, n. 6, p. 782787, 1958 .

SYLVESTER-BRADLEY, R.; AKASAWA, N.; LA TORRACA, S.; MAGALHÃES, F. M. M.; OLIVEIRA, L. A.; PEREIRA, R. M. Levantamento quantitativo de microorganismos solubilizadores de fosfatos na rizosfera de gramíneas e leguminosas forrageiras na Amazônia. Acta Amazonica, Manaus, v. 12, n. 1, p. 15-22, 1982.

TEDESCO, M. J.; GIANELLO, C.; BISSANI, C. A. Análise do solo, plantas e outros materiais. Porto Alegre: UFRGS, 1995. $174 \mathrm{p}$.

WHITELAW, M. A. Growth promotion of plants inoculated with phosphate-solubilizing fungi. Advances in Agronomy, San Diego, v. 69, p. 99-151, 2000. 